\title{
Positive solutions for one-dimensional third-order $p$-Laplacian boundary value problems
}

Yan Sun

\section{"Correspondence:} ysun@shnu.edu.cn Department of Mathematics, Shanghai Normal University, Shanghai, 200234, China

\section{Springer}

\begin{abstract}
In this paper, we give improved results on the existence of positive solutions for the following one-dimensional $p$-Laplacian equation with nonlinear boundary conditions:

$$
\left\{\begin{array}{l}
\left(\phi_{p}\left(y^{\prime \prime}\right)\right)^{\prime}+b(t) g(t, y(t))=0, \quad 0<t<1, \\
\lambda_{1} \phi_{p}(y(0))-\beta_{1} \phi_{p}\left(y^{\prime}(0)\right)=0, \\
\lambda_{2} \phi_{p}(y(1))+\beta_{2} \phi_{p}\left(y^{\prime}(1)\right)=0, \quad y^{\prime \prime}(0)=0,
\end{array}\right.
$$

where $\phi_{p}(s)=|s|^{p-2} s, p>1$. Constructing an available integral operator and combining fixed point index theory, we establish some optimal criteria for the existence of bounded positive solutions. The interesting point of the results is that the term $b(t)$ may be singular at $t=0$ and/or $t=1$. Moreover, the nonlinear term $g(t, y)$ is also allowed to have singularity at $y=0$. In particular, our results extend and unify some known results.
\end{abstract}

MSC: $34 \mathrm{~B} 15$

Keywords: cone; existence; positive solution; maximum principle

\section{Introduction}

In this paper, we investigate the existence of positive solutions for the following onedimensional singular $p$-Laplacian equation with nonlinear boundary conditions:

$$
\left\{\begin{array}{l}
\left(\phi_{p}\left(y^{\prime \prime}\right)\right)^{\prime}+b(t) g(t, y(t))=0, \quad 0<t<1, \\
\lambda_{1} \phi_{p}(y(0))-\beta_{1} \phi_{p}\left(y^{\prime}(0)\right)=0, \\
\lambda_{2} \phi_{p}(y(1))+\beta_{2} \phi_{p}\left(y^{\prime}(1)\right)=0, \quad y^{\prime \prime}(0)=0
\end{array}\right.
$$

where $\phi_{p}(s)=|s|^{p-2} s, p>1, \phi_{q}=\left(\phi_{p}\right)^{-1}, \frac{1}{p}+\frac{1}{q}=1, \lambda_{1}, \lambda_{2}>0, \beta_{1}, \beta_{2} \geq 0, b \in C(0,1), b(t)$ may be singular at $t=0$ and/or $t=1 . g \in C([0,1] \times(0,+\infty))$, and $g(t, y)$ may be singular at $y=0$.

Third-order $p$-Laplacian equations with nonlinear boundary conditions play an important role in both theory and application. They have been paid much more attention to over the years, see [1-9] and the references therein. They are often used to model various phenomena in physics, biology, chemistry, and infections diseases in the positive energy

(c) The Author(s) 2017. This article is distributed under the terms of the Creative Commons Attribution 4.0 International License (http://creativecommons.org/licenses/by/4.0/), which permits unrestricted use, distribution, and reproduction in any medium, provided you give appropriate credit to the original author(s) and the source, provide a link to the Creative Commons license, and indicate if changes were made. 
problem. However, in various situations, including the cases just mentioned above, only positive solutions are meaningful; one refers the reader to see [2-23] for some references along this line. That is why people are particularly interested in studying positive solutions. The existence of positive solutions for $p$-Laplacian equation boundary value problems has been studied by many authors applying various nice methods such as topological degree, the Leray-Schauder continuation theorem, coincidence degree theory and so on (see [2, $4,5,8-24])$.

In [3], He and Ge studied the following nonlinear boundary value problem:

$$
\left\{\begin{array}{l}
\left(\phi_{p}\left(y^{\prime}\right)\right)^{\prime}+q(t) f(t, y(t))=0, \quad 0 \leq t \leq 1 \\
y(0)=g_{1}\left(y^{\prime}(0)\right), \quad y(1)+g_{2}\left(y^{\prime}(1)\right)=0
\end{array}\right.
$$

Their main tool was the fixed point theorem in cones due to Krasnoselskii.

In [2], He studied the existence of double positive solutions for the following nonlinear three-point boundary value problems:

$$
\left\{\begin{array}{l}
\left(\varphi_{p}\left(y^{\prime}\right)\right)^{\prime}+a(t) f(y(t))=0, \quad 0<t<1 \\
y(0)-B_{0}\left(y^{\prime}(\eta)\right)=0, \quad y(1)-B_{1}\left(y^{\prime}(1)\right)=0
\end{array}\right.
$$

and

$$
\left\{\begin{array}{l}
\left(\varphi_{p}\left(y^{\prime}\right)\right)^{\prime}+a(t) f(y(t))=0, \quad 0<t<1 \\
y(0)-B_{0}\left(y^{\prime}(0)\right)=0, \quad y(1)-B_{1}\left(y^{\prime}(\eta)\right)=0 .
\end{array}\right.
$$

He employed a three-functional fixed point theorem due to Avery and Henderson.

Applying the fixed point theorem of cone expansion and compression of norm type, $\mathrm{Su}$ et al. [8] presented the existence of multiple positive solutions of the following nonlinear two-point boundary value problem:

$$
\left\{\begin{array}{l}
\left(\psi_{p}\left(y^{\prime}\right)\right)^{\prime}+a(t) f(y(t))=0, \quad 0<t<1, \\
\alpha \psi_{p}(y(0))-\beta \psi_{p}\left(y^{\prime}(0)\right)=0, \quad \gamma \psi_{p}(y(1))+\delta \psi_{p}\left(y^{\prime}(1)\right)=0 .
\end{array}\right.
$$

Gupta and Trofimchuk [4] established prior bounds and the existence of positive solutions for the following boundary value problem:

$$
\left\{\begin{array}{l}
y^{\prime \prime}(t)=f\left(t, y(t), y^{\prime}(t)\right)+e(t), \quad 0<t<1, \\
y(0)=0, \quad y(1)=\sum_{i=1}^{m-2} a_{i} y\left(\xi_{i}\right)
\end{array}\right.
$$

where $a_{i} \in R, 0<\xi_{1}<\xi_{2}<\cdots<\xi_{m-2}<1$, all $a_{i}$ having the same sign, $\alpha=\sum_{i=1}^{m-1} a_{i}>1$, $\sum_{i=1}^{m-2} a_{i} \xi_{i} \neq 1$.

Feng and Webb [11] considered the following boundary value problem:

$$
\left\{\begin{array}{l}
y^{\prime \prime}(t)=f\left(t, y(t), y^{\prime}(t)\right)+e(t), \quad 0<t<1, \\
y^{\prime}(0)=0, \quad y(1)=\sum_{i=1}^{m-2} a_{i} y\left(\xi_{i}\right)
\end{array}\right.
$$


They presented the existence results with assumptions of nonlinear growth imposed on the nonlinear $\operatorname{term} f$.

Motivated by the results mentioned above, in this paper we study the existence of positive solutions for problem (1.1). We should also assert here that our results are new and generalize the results in [1-3, 9-11].

The rest of the paper is organized as follows. In Section 2, we state some preliminaries and several lemmas in this work. In Section 3, we give the main results as well as some of their proofs. The existence of multiple positive solutions is obtained in Section 4. The existence of infinite positive solutions is presented in Section 5.

\section{Preliminaries}

Definition 2.1 Let $X$ be a real Banach space. A nonempty closed convex set $P \subset X$ is called a cone provided that

(i) $y \in P, \lambda \geq 0$ implies $\lambda y \in P$;

(i) $y \in P,-y \in P$ implies $y=0$.

Definition 2.2 Let $X$ be a real Banach space and $P$ be a cone in $X$. A mapping $\alpha$ is called the nonnegative continuous concave functional on $P$ if $\alpha: P \longrightarrow[0,+\infty)$ is continuous and

$$
\alpha(\lambda t+(1-\lambda) s) \geq \lambda \alpha(t)+(1-\lambda) \alpha(s), \quad s, t \in P, \lambda \in[0,1] .
$$

Definition 2.3 A nonzero solution is said to be a $C[0,1]$ solution of problem (1.1). We indicate a function $y \in C[0,1] \cap C^{3}(0,1)$ satisfying problem (1.1) with $y(t)$ not identically zero on $(0,1) \cdot y(t)$ is said to be a $C^{1}[0,1]$ solution, we indicate that $y^{\prime} \in C[0,1] . y(t)$ is called a positive solution of problem (1.1) if $y(t)$ is a solution of problem (1.1) and $y(t)>0$ for each $t \in(0,1)$.

Let $X=C[0,1]$ be a Banach space with the norm $\|y\|=\sup _{0 \leq t \leq 1}|y(t)|$, and let $K=\{y \in X$ : $y(t) \geq 0,0 \leq t \leq 1\}$. Then $K$ is a positive cone in $X$. Throughout the paper, the partial ordering is always given by $K$. For the concepts and properties of Krein-Kutmann theorems and fixed point index theory, one refers the reader to see [13] and [24]. For $v \in\left(0, \frac{1}{2}\right)$, let

$$
P=\left\{y \in K: \min _{t \in[v, 1-v]} y(t) \geq v\|y\| \text {, and } y(\lambda t+(1-\lambda) s) \geq \lambda y(t)+(1-\lambda) y(s), s, t \in[0,1]\right\} .
$$

Obviously, $P \subset K \subset X$. Denote $P_{r}=\{y \in P:\|y\|<r\}, \partial P_{r}=\{y \in P:\|y\|=r\}, \bar{P}_{r, R}=\{y \in P:$ $r \leq\|y\| \leq R\}$ for any $0<r<R<+\infty$.

Throughout this paper, we suppose that the following conditions hold:

$\left(\mathrm{A}_{1}\right) b \in C((0,1),[0,+\infty)), b(t)$ may be singular at $t=0$ and/or $t=1, b(t) \not \equiv 0$ and

$$
0<\int_{0}^{1} b(s) d s<+\infty
$$

$\left(\mathrm{A}_{2}\right) g(t, y) \in C([0,1] \times(0,+\infty) ;[0,+\infty)), g(t, y)$ may be singular at $y=0$ and for any $0<$ $r<R<+\infty$ such that

$$
\lim _{n \rightarrow+\infty} \sup _{y \in \bar{r}_{r, R}} \int_{E(n)} b(s) g(s, y(s)) d s=0
$$

where $E(n)=\left[0, \frac{1}{n}\right] \cup\left[\frac{n-1}{n}, 1\right]$. 
Remark 2.1 It follows from $\left(\mathrm{A}_{2}\right)$ that

$$
\begin{aligned}
\sup _{y \in \bar{P}_{r, R}} \int_{[0,1]} b(s) g(s, y(s)) d s & =\sup _{y \in \bar{P}_{r, R}} \int_{E(n)} b(s) g(s, y(s)) d s+\sup _{y \in \bar{P}_{r, R}} \int_{\left[\frac{1}{n}, 1-\frac{1}{n}\right]} b(s) g(s, y(s)) d s \\
& <+\infty .
\end{aligned}
$$

$\operatorname{Remark} 2.2$ It is easy to see $\phi_{q}(s)=|s|^{q-2} s$. In fact, from $\frac{1}{p}+\frac{1}{q}=1$, we can obtain

$$
\left(\phi_{q} \phi_{p}\right)(s)=|s|^{p q-2(p+q)+4}|s|^{p+q-4} s=|s|^{p q-(p+q)} s=s .
$$

Remark 2.3 By $\left(\mathrm{A}_{1}\right)$, there exists $t_{0} \in(0,1)$ such that $b\left(t_{0}\right)>0$. Obviously, if $g(t, y)$ is nonsingular at $y=0$, that is, $g \in C([0,1] \times(0,+\infty),[0,+\infty))$, then $\left(\mathrm{A}_{2}\right)$ is satisfied.

Denote

$$
\begin{array}{ll}
g_{0}=\lim _{u \rightarrow 0} \min _{0 \leq t \leq 1} \frac{g(t, u)}{u^{p-1}}, & g_{\infty}=\lim _{u \rightarrow+\infty} \min _{0 \leq t \leq 1} \frac{g(t, u)}{u^{p-1}}, \\
g^{0}=\lim _{u \rightarrow 0} \max _{0 \leq t \leq 1} \frac{g(t, u)}{u^{p-1}}, & g^{\infty}=\lim _{u \rightarrow+\infty} \max _{0 \leq t \leq 1} \frac{g(t, u)}{u^{p-1}} .
\end{array}
$$

Lemma 2.1 Suppose that condition $\left(\mathrm{A}_{1}\right)$ holds. Then there exists a constant $v \in\left(0, \frac{1}{2}\right)$ satisfying

$$
0<\int_{v}^{1-v} b(s) d s<+\infty .
$$

Define a function $f(t)$ on $[v, 1-v]$ given by

$$
\begin{aligned}
f(t) & =\min \left\{\int_{v}^{t} \int_{s}^{\sigma^{*}} \phi_{q}\left(\int_{0}^{r} b(x) d x\right) d r d s, \int_{t}^{1-v} \int_{\sigma^{*}}^{s} \phi_{q}\left(\int_{0}^{r} b(x) d x\right) d r d s\right\}, \\
\sigma^{*} & \in(0,1) .
\end{aligned}
$$

Obviously, $f(t)$ is a continuous and positive function on $[v, 1-v]$ and has its minimum and maximum on $[v, 1-v]$. Therefore, there exist positive constants $m>M>0$ such that $m \leq f(t) \leq M, t \in[v, 1-v]$.

Lemma 2.2 ([13]) Let $X$ be a real Banach space and $P$ be a cone in $X$, with $\Omega(P)$ being a bounded open set in $P, \theta \in \Omega(P)$. Suppose that $T: \bar{\Omega}(P) \rightarrow P$ is a completely continuous operator. If there exists $u_{0} \in P \backslash\{\theta\}$ such that $u-T u \neq \mu u_{0}, \forall u \in \partial \Omega(P), \mu \geq 0$. Then the fixed point index $i(T, \Omega(P), P)=0$.

Lemma 2.3 ([13]) Let $X$ be a Banach space and $P$ be a cone in $X$ with $\Omega(P)$ being a bounded open set in $P, \theta \in \Omega(P)$. Assume that $T: \bar{\Omega}(P) \rightarrow P$ is a completely continuous operator. If $T u \neq \eta u, \forall u \in \partial \Omega(P), \eta \geq 1$, then the fixed point index $i(T, \Omega(P), P)=1$.

Lemma 2.4 ([13]) Let $X$ be a real Banach space and $P$ be a positive cone in $X$. Let $\Omega(P)$ be a bounded open set in $P$ with $\theta \in \Omega(P)$. Suppose that $T: \bar{\Omega}(P) \rightarrow P$ is completely continuous and satisfies the following conditions: 
(i) If $\|T u\|>\|u\|, \forall u \in \partial \Omega(P)$, then the fixed point index $i(T, \Omega(P), P)=0$;

(ii) If $\|T u\|<\|u\|, \theta \in \Omega(P), \forall u \in \partial \Omega(P)$, then the fixed point index $i(T, \Omega(P), P)=1$.

Lemma 2.5 Let $a \in L(0,1)$ be positive. Then the following boundary value problem

$$
\begin{aligned}
& \left(\phi_{p}\left(y^{\prime \prime}\right)\right)^{\prime}+a(t)=0, \quad 0<t<1, \\
& \left\{\begin{array}{l}
\lambda_{1} \phi_{p}(y(0))-\beta_{1} \phi_{p}\left(y^{\prime}(0)\right)=0, \\
\lambda_{2} \phi_{p}(y(1))+\beta_{2} \phi_{p}\left(y^{\prime}(1)\right)=0, \quad y^{\prime \prime}(0)=0,
\end{array}\right.
\end{aligned}
$$

has a unique positive solution which is given explicitly by

$$
y(t)= \begin{cases}\phi_{q}\left(\frac{\beta_{1}}{\lambda_{1}} \phi_{p}\left(\int_{0}^{\sigma^{*}} \phi_{q}\left(\int_{0}^{s} a(r) d r\right) d s\right)\right)+\int_{0}^{t} \int_{s}^{\sigma^{*}} \phi_{q}\left(\int_{0}^{r} a(x) d x\right) d r d s, & 0 \leq t \leq \sigma^{*} \\ \phi_{q}\left(\frac{\beta_{2}}{\lambda_{2}} \phi_{p}\left(\int_{\sigma^{*}}^{1} \phi_{q}\left(\int_{0}^{s} a(r) d r\right) d s\right)\right)+\int_{t}^{1} \int_{\sigma^{*}}^{s} \phi_{q}\left(\int_{0}^{r} a(x) d x\right) d r d s, & \sigma^{*} \leq t \leq 1\end{cases}
$$

Proof It follows from $y^{\prime}(t)=\int_{t}^{\sigma^{*}} \phi_{q}\left(\int_{0}^{r} a(x) d x\right) d r$ and boundary condition (2.2) that $y^{\prime}(0)>$ 0 and $y(0)=\phi_{q}\left(\frac{\beta_{1}}{\lambda_{1}} \phi_{p}\left(y^{\prime}(0)\right)\right)>0, y^{\prime}(1)<0$, with $y(1)=-\phi_{q}\left(\frac{\beta_{2}}{\lambda_{2}} \phi_{p}\left(y^{\prime}(1)\right)\right)<0$. Then there exists a constant $\sigma^{*} \in[0,1]$ such that $y^{\prime}\left(\sigma^{*}\right)=0$.

Integrating (2.1) from 0 to $t$, we get

$$
\phi_{p}\left(y^{\prime \prime}(t)\right)-\phi_{p}\left(y^{\prime \prime}(0)\right)=-\int_{0}^{t} a(s) d s
$$

By making use of $y^{\prime \prime}(0)=0$, we obtain

$$
y^{\prime \prime}(t)=-\phi_{q}\left(\int_{0}^{t} a(s) d s\right)
$$

Integrating (2.3) from $t$ to $\sigma^{*}$, we have

$$
y^{\prime}\left(\sigma^{*}\right)-y^{\prime}(t)=-\int_{t}^{\sigma^{*}} \phi_{q}\left(\int_{0}^{s} a(r) d r\right) d s
$$

Since $y^{\prime}\left(\sigma^{*}\right)=0$, we know that

$$
y^{\prime}(t)=\int_{t}^{\sigma^{*}} \phi_{q}\left(\int_{0}^{s} a(r) d r\right) d s
$$

Thus

$$
y^{\prime}(0)=\int_{0}^{\sigma^{*}} \phi_{q}\left(\int_{0}^{s} a(r) d r\right) d s .
$$

Integrating (2.4) from 0 to $t$, we obtain

$$
y(t)-y(0)=\int_{0}^{t} \int_{s}^{\sigma^{*}} \phi_{q}\left(\int_{0}^{r} a(x) d x\right) d r d s
$$


In view of (2.5) and (2.6) together with boundary conditions (2.2), we have

$$
\begin{aligned}
y(t) & =y(0)+\int_{0}^{t} \int_{s}^{\sigma^{*}} \phi_{q}\left(\int_{0}^{r} a(x) d x\right) d r d s \\
& =\phi_{q}\left(\frac{\beta_{1}}{\lambda_{1}} \phi_{p}\left(y^{\prime}(0)\right)\right)+\int_{0}^{t} \int_{s}^{\sigma^{*}} \phi_{q}\left(\int_{0}^{r} a(x) d x\right) d r d s \\
& =\phi_{q}\left(\frac{\beta_{1}}{\lambda_{1}} \phi_{p}\left(\int_{0}^{\sigma^{*}} \phi_{q}\left(\int_{0}^{s} a(r) d r\right) d s\right)\right)+\int_{0}^{t} \int_{s}^{\sigma^{*}} \phi_{q}\left(\int_{0}^{r} a(x) d x\right) d r d s .
\end{aligned}
$$

Therefore

$$
\begin{aligned}
y(t)= & \phi_{q}\left(\frac{\beta_{1}}{\lambda_{1}} \phi_{p}\left(\int_{0}^{\sigma^{*}} \phi_{q}\left(\int_{0}^{s} a(r) d r\right) d s\right)\right) \\
& +\int_{0}^{t} \int_{s}^{\sigma^{*}} \phi_{q}\left(\int_{0}^{r} a(x) d x\right) d r d s, \quad t \in\left[0, \sigma^{*}\right] .
\end{aligned}
$$

Again, integrating (2.3) from $\sigma^{*}$ to $t$, we see

$$
y^{\prime}(t)-y^{\prime}\left(\sigma^{*}\right)=-\int_{\sigma^{*}}^{t} \phi_{q}\left(\int_{0}^{s} a(r) d r\right) d s .
$$

From $y^{\prime}\left(\sigma^{*}\right)=0$, we have

$$
y^{\prime}(t)=-\int_{\sigma^{*}}^{t} \phi_{q}\left(\int_{0}^{s} a(r) d r\right) d s .
$$

Then

$$
y^{\prime}(1)=-\int_{\sigma^{*}}^{1} \phi_{q}\left(\int_{0}^{s} a(r) d r\right) d s .
$$

Integrating (2.7) from $t$ to 1 , we get

$$
y(1)-y(t)=-\int_{t}^{1} \int_{\sigma^{*}}^{s} \phi_{q}\left(\int_{0}^{r} a(x) d x\right) d r d s .
$$

Thus

$$
y(t)=y(1)+\int_{t}^{1} \int_{\sigma^{*}}^{s} \phi_{q}\left(\int_{0}^{r} a(x) d x\right) d r d s .
$$

Using (2.9) and (2.10) together with boundary conditions (2.2), we obtain

$$
\begin{aligned}
y(t)= & -\phi_{q}\left(\frac{\beta_{2}}{\lambda_{2}} \phi_{p}(y(1))\right)+\int_{t}^{1} \int_{\sigma^{*}}^{s} \phi_{q}\left(\int_{0}^{r} a(x) d x\right) d r d s \\
= & \phi_{q}\left(\frac{\beta_{2}}{\lambda_{2}} \phi_{p}\left(\int_{\sigma^{*}}^{1} \phi_{q}\left(\int_{0}^{s} a(r) d r\right) d s\right)\right) \\
& +\int_{t}^{1} \int_{\sigma^{*}}^{s} \phi_{q}\left(\int_{0}^{r} a(x) d x\right) d r d s, \quad t \in\left[\sigma^{*}, 1\right] .
\end{aligned}
$$

From (2.7) and (2.11) we see that the results of Lemma 2.5 hold. So the proof is complete. 
For $y \in P$, we now define the integral operator $T: P \backslash\{\theta\} \rightarrow P$ by

$$
(T y)(t)=\left\{\begin{array}{cc}
\phi_{q}\left(\frac{\beta_{1}}{\lambda_{1}} \phi_{p}\left(\int_{0}^{\sigma^{*}} \phi_{q}\left(\int_{0}^{s} b(r) g(r, y(r)) d r\right) d s\right)\right) \\
\quad+\int_{0}^{t} \int_{s}^{\sigma^{*}} \phi_{q}\left(\int_{0}^{r} b(x) g(x, y(x)) d x\right) d r d s, \quad 0 \leq t \leq \sigma^{*}, \\
\phi_{q}\left(\frac{\beta_{2}}{\lambda_{2}} \phi_{p}\left(\int_{\sigma^{*}}^{1} \phi_{q}\left(\int_{0}^{s} b(r) g(r, y(r)) d r\right) d s\right)\right) \\
\quad+\int_{t}^{1} \int_{\sigma^{*}}^{s} \phi_{q}\left(\int_{0}^{r} b(x) g(x, y(x)) d x\right) d r d s, \quad \sigma^{*} \leq t \leq 1 .
\end{array}\right.
$$

Lemma 2.6 Suppose that conditions $\left(\mathrm{A}_{1}\right)$ and $\left(\mathrm{A}_{2}\right)$ hold. Then $T: \bar{P}_{r, R} \rightarrow P$ is completely continuous, and the nonzero fixed point $y \in \bar{P}_{r, R}$ of $T$ is a positive solution of problem (1.1).

Proof Firstly, we will show that $T: \bar{P}_{r, R} \rightarrow P$. By a simple computation, for any $y \in P_{r, R}$, we have

$$
\left\{\begin{array}{l}
(T y)^{\prime \prime}(t)=-\phi_{q}\left(\int_{0}^{t} b(s) g(s, y(s)) d s\right) \leq 0 \\
\lambda_{1} \phi_{p}((T y)(0))-\beta_{1} \phi_{p}\left((T y)^{\prime}(0)\right)=0 \\
\lambda_{2} \phi_{p}((T y)(1))+\beta_{2} \phi_{p}\left((T y)^{\prime}(1)\right)=0, \quad(T y)^{\prime \prime}(0)=0
\end{array}\right.
$$

that is, $(T y)(t)$ is a nonnegative concave function. We suppose that $\tau=\inf \{\zeta \in[0,1]$ : $\left.\sup _{0 \leq t \leq 1} y(t)=y(\zeta)\right\}$. Now we shall make the following discussions.

Case (1) $\tau \in[0, v]$. It follows from the concavity of $y(t)$ that each point on chord between $(\tau, y(\tau))$ and $(1, y(1))$ is below the graph of $y(t)$. Thus, for any $t \in[\nu, 1-\nu]$, we get

$$
\begin{aligned}
y(t) & \geq y(\tau)+\frac{y(1)-y(\tau)}{1-\tau}(t-\tau) \\
& \geq \min _{t \in[v, 1-\nu]}\left[y(\tau)+\frac{y(1)-y(\tau)}{1-\tau}(t-\tau)\right]=y(\tau)+\frac{y(1)-y(\tau)}{1-\tau}(1-v-\tau) \\
& =\frac{1-v-\tau}{1-\tau} y(1)+\frac{v}{1-\tau} y(\tau) \geq v y(\tau),
\end{aligned}
$$

which implies $y(t) \geq v\|y\|$ for $t \in[v, 1-v]$.

Case (2) $\tau \in[\nu, 1-v]$. If $t \in[\nu, \tau]$, we obtain

$$
\begin{aligned}
y(t) & \geq y(\tau)+\frac{y(\tau)-y(0)}{\tau}(t-\tau) \geq \min _{t \in[v, \tau]}\left[y(\tau)+\frac{y(\tau)-y(0)}{\tau}(t-\tau)\right] \\
& =\frac{v}{\tau} y(\tau)+\frac{\tau-v}{\tau} y(0) \geq \frac{v}{\tau} y(\tau) \geq v y(\tau),
\end{aligned}
$$

which implies $y(t) \geq v\|y\|$ for $t \in[v, \tau]$.

If $t \in[\tau, 1-v]$, we get

$$
\begin{aligned}
y(t) & \geq y(\tau)+\frac{y(1)-y(\tau)}{1-\tau}(t-\tau) \\
& \geq \min _{t \in[\tau, 1-v]}\left[y(\tau)+\frac{y(1)-y(\tau)}{1-\tau}(t-\tau)\right]=y(\tau)+\frac{y(1)-y(\tau)}{1-\tau}(1-v-\tau) \\
& =\frac{1-v-\tau}{1-\tau} y(1)+\frac{v}{1-\tau} y(\tau) \geq \frac{v}{1-\tau} y(\tau) \geq v y(\tau),
\end{aligned}
$$

which implies $y(t) \geq v\|y\|$ for $t \in[\tau, 1-v]$. Therefore, $y(t) \geq v\|y\|$ for $t \in[v, 1-v]$. 
Case (3) $\tau \in[1-v, 1]$. By the same argument, for $t \in[v, 1-v]$, we have

$$
\begin{aligned}
y(t) & \geq y(\tau)+\frac{y(\tau)-y(0)}{\tau}(t-\tau) \\
& \geq \min _{t \in[v, 1-v]}\left[y(\tau)+\frac{y(\tau)-y(0)}{\tau}(t-\tau)\right] \\
& =y(\tau)+\frac{y(\tau)-y(0)}{\tau}(v-\tau) \\
& \geq \frac{v}{\tau} y(\tau)+\frac{\tau-v}{\tau} y(0) \geq \frac{v}{\tau} y(\tau) \geq v y(\tau),
\end{aligned}
$$

which implies $y(t) \geq v\|y\|$ for $t \in[v, 1-v]$. Using the above discussions, one can get $y(t) \geq$ $v\|y\|$ for $t \in[v, 1-v]$. Thus $T P_{r, R} \subset P$.

Next, for any $0<r<R<+\infty$, we will show

$$
\sup _{y \in \partial \bar{P}_{r, R}} \int_{0}^{1} b(s) g(s, y(s)) d s<+\infty
$$

which implies that $T: P \backslash\{0\} \rightarrow P$ is well defined.

From $\left(\mathrm{A}_{2}\right)$ and Remark 2.1, for any $0<r<R<+\infty$, there exists a natural number $k$ such that

$$
\sup _{y \in \bar{P}_{r, R}} \int_{E(k)} b(s) g(s, y(s)) d s<1 .
$$

Thus

$$
\sup _{y \in \bar{P}_{r, R}} \int_{v}^{1-v} b(s) g(s, y(s)) d s<\sup _{y \in \bar{P}_{r, R}} \int_{0}^{1} b(s) g(s, y(s)) d s<+\infty .
$$

For any $y \in \partial P_{r}$, let $y\left(t_{0}\right)=\max _{t \in[0,1]}|y(t)|=r, t_{0} \in[0,1]$. Denote

$$
\chi_{E[a, b]}(t)= \begin{cases}1, & t \in[a, b], \\ 0, & t \notin[a, b]\end{cases}
$$

is the eigenvalue function of the set $E[a, b]=\{t: a \leq t \leq b\}$. It follows from the concavity of $y(t)$ on $[0,1]$ that

$$
\begin{aligned}
y(t) & \geq \frac{r t}{t_{0}} \chi_{E\left[0, t_{0}\right]}(t)+\frac{r}{1-t_{0}}(1-t) \chi_{E\left[t_{0}, 1\right]}(t) \\
& \geq r t \chi_{E\left[0, t_{0}\right]}(t)+r(1-t) \chi_{E\left[t_{0}, 1\right]}(t) .
\end{aligned}
$$

Consequently, from (2.14), we have

$$
\frac{r}{R} \leq y(t) \leq r<R \quad \text { for any } t \in\left[\frac{1}{k}, \frac{k-1}{k}\right]
$$

Denote

$$
\bar{G}=\max \left\{g(t, y):(t, y) \in\left[\frac{1}{k}, \frac{k-1}{k}\right] \times\left[\frac{r}{k}, R\right]\right\} .
$$


Sun Advances in Difference Equations ( 2017) 2017:95

Page 9 of 24

It follows from $\left(\mathrm{A}_{1}\right)$ and $\left(\mathrm{A}_{2}\right)$ with $(2.13)-(2.16)$ that

$$
\begin{aligned}
\sup _{y \in \partial \bar{P}_{r, R}} \int_{0}^{1} b(s) g(s, y(s)) d s \leq & \sup _{y \in \partial \bar{P}_{r, R}} \int_{E(k)} b(s) g(s, y(s)) d s \\
& +\sup _{y \in \partial \bar{P}_{r, R}} \int_{\frac{1}{k}}^{\frac{k-1}{k}} b(s) g(s, y(s)) d s \\
\leq & 1+\bar{G} \int_{0}^{1} b(s) d s<+\infty .
\end{aligned}
$$

Thus (2.12) holds. It also implies that $T: \bar{P}_{r, R} \rightarrow P$ is well defined and $T(Q)$ is uniformly bounded for any bounded set $Q \subset \bar{P}_{r, R}$.

Now, we prove that for any $0<r<R<+\infty, T\left(\bar{P}_{r, R}\right)$ is equicontinuous. In fact, from $\left(\mathrm{A}_{2}\right)$, for any $\varepsilon>0$, there exists a natural number $k_{0}$ such that

$$
\sup _{y \in \bar{P}_{r, R}} \int_{E\left(k_{0}\right)} b(s) g(s, y(s)) d s<\phi_{p}\left(\frac{\varepsilon}{4}\right) .
$$

Let

$$
\widehat{G}=\max _{k_{0} \in N}\left\{\phi_{q}\left(\int_{\frac{1}{k_{0}}}^{\frac{k_{0}-1}{k_{0}}} b(x) g(x, y(x)) d x\right):(t, y) \in\left[\frac{1}{k_{0}}, \frac{k_{0}-1}{k_{0}}\right] \times\left[\frac{r}{k_{0}}, R\right]\right\} .
$$

Then

$$
\int_{\frac{1}{k_{0}}}^{\frac{k_{0}-1}{k_{0}}} b(x) g(x, y(x)) d x \leq \phi_{p}(\widehat{G}) .
$$

For the above $\varepsilon>0$, take $0<\delta<\frac{\varepsilon}{2 \widehat{G}+\varepsilon}$ such that for any $t^{\prime}, t^{\prime \prime} \in[0,1]$ satisfying $\left|t^{\prime}-t^{\prime \prime}\right|<\delta$ and for any $s \in\left[\frac{1}{k_{0}}, \frac{k_{0}-1}{k_{0}}\right]$, we have

$$
\begin{aligned}
\left|(T y)\left(t^{\prime}\right)-(T y)\left(t^{\prime \prime}\right)\right|= & \left|\int_{t^{\prime}}^{t^{\prime \prime}} \int_{s}^{\sigma^{*}} \phi_{q}\left(\int_{0}^{r} b(x) g(x, y(x)) d x\right) d r d s\right| \\
\leq & \left|\int_{t^{\prime}}^{t^{\prime \prime}} \int_{s}^{\sigma^{*}} \phi_{q}\left(\int_{\frac{1}{k_{0}}}^{\frac{k_{0}-1}{k_{0}}} b(x) g(x, y(x)) d x\right) d r d s\right| \\
& +2\left|\int_{t^{\prime}}^{t^{\prime \prime}} \int_{s}^{\sigma^{*}} \phi_{q}\left(\int_{E\left(k_{0}\right)} b(x) g(x, y(x)) d x\right) d r d s\right| \\
\leq & \left|\int_{t^{\prime}}^{t^{\prime \prime}} \int_{s}^{\sigma^{*}} \widehat{G} d r d s\right|+2\left|\int_{t^{\prime}}^{t^{\prime \prime}} \int_{s}^{\sigma^{*}} \frac{\varepsilon}{4} d r d s\right| \\
= & \widehat{G}\left|-\frac{1}{2}\left(\sigma^{*}-s\right)^{2}\right| t_{t^{\prime}}^{t^{\prime \prime}}\left|+\frac{\varepsilon}{2}\right|-\left.\frac{1}{2}\left(\sigma^{*}-s\right)^{2}\right|_{t^{\prime}} ^{t^{\prime \prime}} \mid \\
= & \widehat{G}\left|\frac{1}{2}\left(t^{\prime}-t^{\prime \prime}\right)\left(2 \sigma^{*}-t^{\prime}-t^{\prime \prime}\right)\right|+\frac{\varepsilon}{2}\left|\frac{1}{2}\left(t^{\prime}-t^{\prime \prime}\right)\left(2 \sigma^{*}-t^{\prime}-t^{\prime \prime}\right)\right| \\
< & \widehat{G} \cdot \frac{\varepsilon}{2 \widehat{G}+\varepsilon}+\frac{\varepsilon}{2} \cdot \frac{\varepsilon}{2 \widehat{G}+\varepsilon} \leq \frac{\varepsilon}{2}+\frac{\varepsilon}{2}=\varepsilon .
\end{aligned}
$$


This implies that $T\left(\bar{P}_{r, R}\right)$ is equicontinuous. Thus, by the Ascoli-Arzela theorem, we know that $T: \bar{P}_{r, R} \rightarrow P$ is compact.

Finally we show that $T: \bar{P}_{r, R} \rightarrow P$ is continuous. Suppose that $y_{n}, y_{0} \in \bar{P}_{r, R}$ and $\| y_{n}-$ $y_{0} \| \rightarrow 0(n \rightarrow \infty)$. Then $r \leq\left\|y_{n}\right\| \leq R$ and $r \leq\left\|y_{0}\right\| \leq R$. For any $\varepsilon>0$, by making use of $\left(\mathrm{A}_{2}\right)$, there exists a natural number $n_{0}>0$ such that

$$
\begin{gathered}
\sup _{y \in \bar{P}_{r, R}}\left\{\phi_{q}\left(\frac{\beta_{i}}{\lambda_{i}} \phi_{p}\left(\int_{0}^{1} \phi_{q}\left(\int_{E\left(n_{0}\right)} b(r) g(r, y(r)) d r\right) d s\right)\right)\right. \\
\left.+\int_{0}^{1} \int_{0}^{1} \phi_{q}\left(\int_{E\left(n_{0}\right)} b(x) g(x, y(x)) d x\right) d r d s\right\} \\
<\frac{\varepsilon}{4} \quad \text { for } i=1,2 .
\end{gathered}
$$

On the other hand, from (2.15), we have

$$
\frac{r}{n_{0}} \leq y_{n}(t) \leq R, \quad t \in\left[\frac{1}{n_{0}}, \frac{n_{0}-1}{n_{0}}\right]
$$

Since $g(t, y)$ is uniformly continuous on $\left[\frac{1}{n_{0}}, \frac{n_{0}-1}{n_{0}}\right] \times\left[\frac{r}{n_{0}}, R\right]$, we have

$$
\lim _{n \rightarrow+\infty}\left|g\left(s, y_{n}(s)\right)-g\left(s, y_{0}(s)\right)\right|=0
$$

holds uniformly on $s \in\left[\frac{1}{n_{0}}, \frac{n_{0}-1}{n_{0}}\right]$. Then the Lebesgue dominated convergence theorem yields that

$$
\int_{\frac{1}{n_{0}}}^{\frac{n_{0}-1}{n_{0}}} b(s)\left|g\left(s, y_{n}(s)\right)-g\left(s, y_{0}(s)\right)\right| d s \rightarrow 0 \quad(n \rightarrow \infty) .
$$

Thus, for the above $\varepsilon>0$, there exists a natural number $N$, for any $n>N$, we have

$$
\begin{aligned}
& \phi_{q}\left(\frac{\beta_{i}}{\lambda_{i}} \phi_{p}\left(\int_{0}^{1} \phi_{q}\left(\int_{\frac{1}{n_{0}}}^{\frac{n_{0}-1}{n_{0}}} b(r)\left|g\left(r, y_{n}(r)\right)-g\left(r, y_{0}(r)\right)\right| d r\right) d s\right)\right) \\
& \quad+\int_{0}^{1} \int_{0}^{1} \phi_{q}\left(\int_{\frac{1}{n_{0}}}^{\frac{n_{0}-1}{n_{0}}} b(x)\left|g\left(x, y_{n}(x)\right)-g\left(x, y_{0}(x)\right)\right| d x\right) d r d s \\
& <\frac{\varepsilon}{2} \quad \text { for } i=1,2 .
\end{aligned}
$$

It follows from (2.18) and (2.19) that for any $n>N$, we see

$$
\begin{aligned}
\left\|T y_{n}-T y_{0}\right\| \leq & 2 \phi_{q}\left(\frac{\beta_{i}}{\lambda_{i}} \phi_{p}\left(\int_{0}^{1} \phi_{q}\left(\int_{E\left(n_{0}\right)} b(r)\left|g\left(r, y_{n}(r)\right)-g\left(r, y_{0}(r)\right)\right| d r\right) d s\right)\right) \\
& +2 \int_{0}^{1} \int_{0}^{1} \phi_{q}\left(\int_{E\left(n_{0}\right)} b(x)\left|g\left(x, y_{n}(x)\right)-g\left(x, y_{0}(x)\right)\right| d x\right) d r d s \\
& +\phi_{q}\left(\frac{\beta_{i}}{\lambda_{i}} \phi_{p}\left(\int_{0}^{1} \phi_{q}\left(\int_{\frac{1}{n_{0}}}^{\frac{n_{0}-1}{n_{0}}} b(r)\left|g\left(r, y_{n}(r)\right)-g\left(r, y_{0}(r)\right)\right| d r\right) d s\right)\right)
\end{aligned}
$$




$$
\begin{aligned}
& \quad+\int_{0}^{1} \int_{0}^{1} \phi_{q}\left(\int_{\frac{1}{n_{0}}}^{\frac{n_{0}-1}{n_{0}}} b(x)\left|g\left(x, y_{n}(x)\right)-g\left(x, y_{0}(x)\right)\right| d x\right) d r d s \\
& <2 \cdot \frac{\varepsilon}{4}+\frac{\varepsilon}{2}=\varepsilon \quad \text { for } i=1,2 .
\end{aligned}
$$

This implies that $T: \bar{P}_{r, R} \rightarrow P$ is continuous. Thus $T: \bar{P}_{r, R} \rightarrow P$ is completely continuous.

Obviously, if $T$ has a nonzero fixed point $y \neq 0$, then $y \in C[0,1] \cap C^{3}(0,1)$ and satisfies problem (1.1). On the other hand, by the maximum principle, we see that $y(t)>0, t \in(0,1)$. Hence $y$ is a positive solution of problem (1.1). This completes the proof.

\section{The existence of at least one positive solution}

For convenience, we let $\omega^{*}$ and $\omega_{*}$ be positive constants satisfying $\omega^{*}>\frac{1}{m}$ and

$$
0<\omega_{*}=\frac{1}{\max \left\{\left(1+\phi_{q}\left(\frac{\beta_{1}}{\lambda_{1}}\right)\right) \phi_{q}\left(2 \int_{0}^{1} b(r) d r\right),\left(1+\phi_{q}\left(\frac{\beta_{2}}{\lambda_{2}}\right)\right) \phi_{q}\left(2 \int_{0}^{1} b(r) d r\right)\right\}} .
$$

Thus, for any positive constants $l, L, \hat{r}, \widehat{R}$ satisfying $\omega^{*}<l<+\infty, 0<L<\omega_{*}$ and $0<\hat{r}<\widehat{R}<$ $+\infty$, we have the following theorems.

Theorem 3.1 Suppose that conditions $\left(\mathrm{A}_{1}\right)$ and $\left(\mathrm{A}_{2}\right)$ hold. In addition, assume that

$$
\begin{aligned}
& (l \hat{r})^{p-1} \leq g(t, y), \quad(t, y) \in[0,1] \times[v \hat{r}, \hat{r}] \quad \text { and } \\
& g(t, y) \leq(L \widehat{R})^{p-1}, \quad(t, y) \in[0,1] \times(0, \widehat{R}] .
\end{aligned}
$$

Then boundary value problem (1.1) has at least one positive solution $\hat{y}$ satisfying $\hat{r} \leq \hat{y} \leq \widehat{R}$.

Proof From the first part of (3.1), $g(t, y) \geq(l \hat{r})^{p-1}$, for $(t, y) \in[0,1] \times[v \hat{r}, \hat{r}]$, let $\psi \equiv 1$. We prove that

$$
y \neq T y+\mu \psi \quad \text { for } y \in \partial P_{\hat{r}} \text { and } \mu \geq 0 .
$$

If not, there exist $y_{0} \in \partial P_{\hat{r}}$ and $\mu_{0} \geq 0$ such that $y_{0}=T y_{0}+\mu_{0} \psi$. Let $\mu^{*}=\min _{t \in[0,1]} y_{0}(t)$. Then, for $t \in\left(0, \sigma^{*}\right)$, we have

$$
\begin{aligned}
y_{0}(t)= & T y_{0}(t)+\mu_{0} \\
= & \phi_{q}\left(\frac{\beta_{1}}{\lambda_{1}} \phi_{p}\left(\int_{0}^{\sigma^{*}} \phi_{q}\left(\int_{0}^{s} b(r) g\left(r, y_{0}(r)\right) d r\right) d s\right)\right) \\
& +\int_{0}^{t} \int_{s}^{\sigma^{*}} \phi_{q}\left(\int_{0}^{r} b(x) g\left(x, y_{0}(x)\right) d x\right) d r d s+\mu_{0} \\
\geq & \int_{0}^{t} \int_{s}^{\sigma^{*}} \phi_{q}\left(\int_{0}^{r} b(x) g\left(x, y_{0}(x)\right) d x\right) d r d s+\mu_{0} \\
\geq & (l \hat{r}) \int_{0}^{t} \int_{s}^{\sigma^{*}} \phi_{q}\left(\int_{0}^{r} b(x) d x\right) d r d s+\mu_{0} \\
\geq & (l \hat{r}) \int_{v}^{t} \int_{s}^{\sigma^{*}} \phi_{q}\left(\int_{0}^{r} b(x) d x\right) d r d s+\mu_{0} \\
\geq & (l \hat{r}) f(t)+\mu_{0} \geq(l \hat{r}) m+\mu_{0}>\hat{r}=\left\|y_{0}\right\|>\mu^{*} .
\end{aligned}
$$


On the other hand, for $t \in\left[\sigma^{*}, 1\right]$, by the same argument, we have

$$
\begin{aligned}
y_{0}(t) & =T y_{0}(t)+\mu_{0} \\
& \geq \int_{t}^{1} \int_{\sigma^{*}}^{s} \phi_{q}\left(\int_{0}^{r} b(x) g(x, y(x)) d x\right) d r d s+\mu_{0} \\
& \geq(l \hat{r}) \int_{t}^{1} \int_{\sigma^{*}}^{s} \phi_{q}\left(\int_{0}^{r} b(x) d x\right) d r d s+\mu_{0} \\
& \geq(l \hat{r}) \int_{t}^{1-\nu} \int_{\sigma^{*}}^{s} \phi_{q}\left(\int_{0}^{r} b(x) d x\right) d r d s+\mu_{0} \\
& \geq(l \hat{r}) f(t)+\mu_{0} \geq(l \hat{r}) m+\mu_{0}>\hat{r}=\|y\|>\mu^{*} .
\end{aligned}
$$

From the above discussions, we see $y_{0}>\mu^{*}$, which implies $\mu^{*}>\mu^{*}$, a contradiction with the definition of $\mu^{*}$. Thus, we know that (3.2) holds. It follows from Lemma 2.2 that

$$
i\left(T, P_{\hat{r}}, P\right)=0 .
$$

Without loss of generality, we may assume that $T$ has no fixed point on $\partial P_{\hat{r}}$ and $\partial P_{\widehat{R}}$. Now we prove that

$$
T y \neq \eta y, \quad \forall y \in \partial P_{\widehat{R}}, \eta \geq 1 .
$$

In fact, if not, there exist $y_{1} \in \partial P_{\widehat{R}}$ and $\eta_{1} \geq 1$ such that $T y_{1}=\eta_{1} y_{1}$. Let $y_{1}^{*}(t)=\min \left\{y_{1}(t), \widehat{R}\right\}$, thus $y_{1}^{*} \in \partial P_{\widehat{R}}$. Thus, for $t \in\left(0, \sigma^{*}\right)$, we have

$$
\begin{aligned}
y_{1}= & \frac{1}{\eta_{1}} T y_{1} \\
= & \frac{1}{\eta_{1}} \phi_{q}\left(\frac{\beta_{1}}{\lambda_{1}} \phi_{p}\left(\int_{0}^{\sigma^{*}} \phi_{q}\left(\int_{0}^{s} b(r) g\left(r, y_{1}(r)\right) d r\right) d s\right)\right) \\
& +\frac{1}{\eta_{1}} \int_{0}^{t} \int_{s}^{\sigma^{*}} \phi_{q}\left(\int_{0}^{r} b(x) g\left(x, y_{1}(x)\right) d x\right) d r d s \\
\leq & \phi_{q}\left(\frac{\beta_{1}}{\lambda_{1}} \int_{0}^{1} b(r) g\left(r, y_{1}(r)\right) d r\right)+\phi_{q}\left(\int_{0}^{1} b(r) g\left(r, y_{1}(r)\right) d r\right) \\
= & \left(1+\phi_{q}\left(\frac{\beta_{1}}{\lambda_{1}}\right)\right) \int_{0}^{1} b(r) g\left(r, y_{1}(r)\right) d r \\
\leq & L \widehat{R}\left(1+\phi_{q}\left(\frac{\beta_{1}}{\lambda_{1}}\right)\right) \phi_{q}\left(\int_{0}^{1} b(r) d r\right) \\
< & \omega_{*} \cdot \widehat{R} \cdot \frac{1}{\omega_{*}}=\widehat{R}=\left\|y_{1}^{*}\right\| .
\end{aligned}
$$

On the other hand, for $t \in\left[\sigma^{*}, 1\right]$, by the same argument, we have

$$
\begin{aligned}
y_{1} & =\frac{1}{\eta_{1}} T y_{1} \\
& =\frac{1}{\eta_{1}}\left(\phi_{q}\left(\frac{\beta_{2}}{\lambda_{2}} \phi_{p}\left(\int_{\sigma^{*}}^{1} \phi_{q}\left(\int_{0}^{s} b(r) g\left(r, y_{1}(r)\right) d r\right) d s\right)\right)\right.
\end{aligned}
$$




$$
\begin{aligned}
& \left.+\int_{t}^{1} \int_{\sigma^{*}}^{s} \phi_{q}\left(\int_{0}^{r} b(x) g\left(x, y_{1}(x)\right) d x\right) d r d s\right) \\
\leq & \phi_{q}\left(\frac{\beta_{2}}{\lambda_{2}} \int_{0}^{1} b(r) g\left(r, y_{1}(r)\right) d r\right)+\phi_{q}\left(\int_{0}^{1} b(r) g\left(r, y_{1}(r)\right) d r\right) \\
= & \left(1+\phi_{q}\left(\frac{\beta_{2}}{\lambda_{2}}\right)\right) \int_{0}^{1} b(r) g\left(r, y_{1}(r)\right) d r \\
\leq & L \widehat{R}\left(1+\phi_{q}\left(\frac{\beta_{2}}{\lambda_{2}}\right)\right) \phi_{q}\left(\int_{0}^{1} b(r) d r\right) \\
< & \omega_{*} \cdot \widehat{R} \cdot \frac{1}{\omega_{*}}=\widehat{R}=\left\|y_{1}^{*}\right\| .
\end{aligned}
$$

The above discussions imply that $\left\|y_{1}^{*}\right\|<\left\|y_{1}^{*}\right\|$, a contradiction with the definition of $y_{1}^{*}$. Therefore (3.4) holds. It follows from Lemma 2.3 that

$$
i\left(T, P_{\widehat{R}}, P\right)=1
$$

From (3.3) and (3.5) together with the properties of fixed point index, we have

$$
i\left(T, P_{\widehat{r}, \widehat{R}}, P\right)=i\left(T, P_{\widehat{R}}, P\right)-i\left(T, P_{\hat{r}}, P\right)=1-0=1
$$

Thus $T$ has at least one positive fixed point $\hat{y}$ in $P_{\hat{r}, \widehat{R}}$, which means that problem (1.1) has at least one positive solution $\hat{y}$ satisfying $\hat{r} \leq \hat{y} \leq \widehat{R}$. This completes the proof.

Theorem 3.2 Suppose that conditions $\left(\mathrm{A}_{1}\right)$ and $\left(\mathrm{A}_{2}\right)$ hold. In addition, assume that the following conditions hold:

$\left(\mathrm{H}_{1}\right) \quad 0<g^{0}<\left(\frac{\omega_{*}}{2}\right)^{p-1}$,

$\left(\mathrm{H}_{2}\right)\left(\frac{\omega^{*}}{v}\right)^{p-1}<g_{\infty}<+\infty$.

Then problem (1.1) has at least one positive solution.

Proof From $\left(\mathrm{H}_{1}\right)$, for any $0<\varepsilon<\left(\frac{\omega_{*}}{2}\right)^{p-1}-g^{0}$, there exists a sufficiently small positive constant $r_{1}$ such that $0<y \leq r_{1}$, for any $t \in[0,1]$, we have

$$
g(t, y) \leq y^{p-1}\left(g_{0}+\varepsilon\right)<y^{p-1}\left(\frac{\omega_{*}}{2}\right)^{p-1}, \quad(t, y) \in[0,1] \times\left(0, r_{1}\right]
$$

Since $y^{\prime}\left(\sigma^{*}\right)=0$, we find that

$$
\begin{gathered}
\phi_{q}\left(\frac{\beta_{1}}{\lambda_{1}} \phi_{p}\left(\int_{0}^{\sigma^{*}} \phi_{q}\left(\int_{0}^{s} b(r) g(r, y(r)) d r\right) d s\right)\right) \\
\quad+\int_{0}^{\sigma^{*}} \int_{s}^{\sigma^{*}} \phi_{q}\left(\int_{0}^{r} b(x) g(x, y(x)) d x\right) d r d s \\
=\phi_{q}\left(\frac{\beta_{2}}{\lambda_{2}} \phi_{p}\left(\int_{\sigma^{*}}^{1} \phi_{q}\left(\int_{0}^{s} b(r) g(r, y(r)) d r\right) d s\right)\right) \\
+\int_{\sigma^{*}}^{1} \int_{\sigma^{*}}^{s} \phi_{q}\left(\int_{0}^{r} b(x) g(x, y(x)) d x\right) d r d s .
\end{gathered}
$$


Thus, from (3.6) and (3.7), we obtain

$$
\begin{aligned}
\|T y\|= & (T y)\left(\sigma^{*}\right) \\
\leq & \phi_{q}\left(\frac{\beta_{i}}{\lambda_{i}} \phi_{p}\left(\int_{0}^{1} \phi_{q}\left(\int_{0}^{1} b(r) g(r, y(r)) d r\right) d s\right)\right) \\
& +\int_{0}^{1} \int_{0}^{1} \phi_{q}\left(\int_{0}^{1} b(x) g(x, y(x)) d x\right) d r d s \\
= & \phi_{q}\left(\frac{\beta_{i}}{\lambda_{i}} \int_{0}^{1} b(r) g(r, y(r)) d r\right)+\phi_{q}\left(\int_{0}^{1} b(r) g(r, y(r)) d r\right) \\
\leq & \left(1+\phi_{q}\left(\frac{\beta_{i}}{\lambda_{i}}\right)\right) \phi_{q}\left(\int_{0}^{1} b(r) g(r, y(r)) d r\right) \\
< & \frac{\omega_{*} r_{1}}{2}\left(1+\phi_{q}\left(\frac{\beta_{i}}{\lambda_{i}}\right)\right) \phi_{q}\left(\int_{0}^{1} b(r) d r\right) \\
< & \frac{\omega_{*} r_{1}}{2} \cdot \omega_{*}=\frac{r_{1}}{2}<r_{1}=\|y\| \text { for } i=1,2 .
\end{aligned}
$$

Indeed, in view of Lemma 2.4, we deduce

$$
i\left(T, P_{r_{1}}, P\right)=1 \text {. }
$$

Next from $\left(\mathrm{H}_{2}\right)$, for any $0<\varepsilon<g_{\infty}-\left(\frac{\omega^{*}}{v}\right)^{p-1}$, there exists a sufficiently large positive constant $R^{*}$ such that for any $y \geq R^{*}$, we have

$$
g(t, y) \geq\left(g_{\infty}-\varepsilon\right) y^{p-1}, \quad y \geq R^{*}, t \in[0,1] .
$$

Choose $R_{1}=\max \left\{2 r_{1}, \frac{R^{*}}{v}\right\}$, and let $\varphi(t) \equiv 1, t \in[0,1]$. Then $R_{1}>r_{1}$ and $\varphi \in \partial P_{1}$. Obviously, $\varphi(t) \in P_{R_{1}} \backslash\{\theta\}$.

In the following we prove that

$$
y \neq T y+\mu \varphi \quad \text { for } y \in \partial P_{R_{1}} \text { and } \mu \geq 0 .
$$

Otherwise, there exist $y_{0} \in \partial P_{R_{1}}$ and $\mu_{0}>0$ such that $y_{0}=T y_{0}+\mu_{0} \varphi$. Let $\xi=\min \left\{y_{0}(t): t \in\right.$ $[v, 1-v]\}$ and notice that for any $t \in[v, 1-v]$, we have $\min _{t \in[v, 1-v]} y(t) \geq v\left\|y_{0}\right\|=v R_{1} \geq R^{*}$.

Consequently, from (3.9), for any $t \in[\nu, 1-\nu]$ and $0<t<\sigma^{*}$, we have

$$
\begin{aligned}
y_{0}(t)= & T y_{0}(t)+\mu_{0} \varphi(t) \\
= & \phi_{q}\left(\frac{\beta_{1}}{\lambda_{1}} \phi_{p}\left(\int_{0}^{\sigma^{*}} \phi_{q}\left(\int_{0}^{s} b(r) g\left(r, y_{0}(r)\right) d r\right) d s\right)\right) \\
& +\int_{0}^{t} \int_{s}^{\sigma^{*}} \phi_{q}\left(\int_{0}^{r} b(x) g\left(x, y_{0}(x)\right) d x\right) d r d s+\mu_{0} \\
\geq & \int_{0}^{t} \int_{s}^{\sigma^{*}} \phi_{q}\left(\int_{0}^{r} b(x) g\left(x, y_{0}(x)\right) d x\right) d r d s+\mu_{0} \\
\geq & \int_{0}^{t} \int_{s}^{\sigma^{*}} \phi_{q}\left(\int_{0}^{r} b(x)\left(\frac{\omega^{*} y_{0}(x)}{v}\right)^{p-1} d x\right) d r d s+\mu_{0}
\end{aligned}
$$




$$
\begin{aligned}
& \geq \frac{\omega^{*} v\left\|y_{0}\right\|}{v} \int_{v}^{t} \int_{s}^{\sigma^{*}} \phi_{q}\left(\int_{0}^{r} b(x) d x\right) d r d s+\mu_{0} \\
& \geq \omega^{*} \cdot \xi \cdot f(t)+\mu_{0} \geq \omega^{*} \cdot \xi \cdot m+\mu_{0}>\xi+\mu_{0}>\xi .
\end{aligned}
$$

On the other hand, by the same argument, for $t \in\left[\sigma^{*}, 1\right]$ and $t \in[v, 1-v]$, from (3.9) and (3.7), we have

$$
\begin{aligned}
y_{0}(t)= & T y_{0}(t)+\mu_{0} \varphi(t) \\
= & \phi_{q}\left(\frac{\beta_{2}}{\lambda_{2}} \phi_{p}\left(\int_{\sigma^{*}}^{1} \phi_{q}\left(\int_{0}^{s} b(r) g\left(r, y_{0}(r)\right) d r\right) d s\right)\right) \\
& +\int_{t}^{1} \int_{\sigma^{*}}^{s} \phi_{q}\left(\int_{0}^{r} b(x) g\left(x, y_{0}(x)\right) d x\right) d r d s+\mu_{0} \\
\geq & \int_{t}^{1} \int_{\sigma^{*}}^{s} \phi_{q}\left(\int_{0}^{r} b(x) g\left(x, y_{0}(x)\right) d x\right) d r d s+\mu_{0} \\
\geq & \int_{t}^{1} \int_{\sigma^{*}}^{s} \phi_{q}\left(\int_{0}^{r} b(x)\left(\frac{\omega^{*} y_{0}(x)}{v}\right)^{p-1} d x\right) d r d s+\mu_{0} \\
\geq & \frac{\omega^{*} v\left\|y_{0}\right\|}{v} \int_{t}^{1-v} \int_{\sigma^{*}}^{s} \phi_{q}\left(\int_{0}^{r} b(x) d x\right) d r d s+\mu_{0} \\
\geq & \omega^{*} \cdot \xi \cdot f(t)+\mu_{0} \geq \omega^{*} \cdot \xi \cdot m+\mu_{0}>\xi+\mu_{0}>\xi .
\end{aligned}
$$

From (3.11) and (3.12), we easily obtain $\xi>\xi$, a contradiction with the definition of $\xi$. From the above discussion we see that (3.10) holds. It follows from Lemma 2.2 that

$$
i\left(T, P_{R_{1}}, P\right)=0 \text {. }
$$

From (3.8) and (3.13) together with the properties of fixed point index, we have

$$
i\left(T, P_{r_{1}, R_{1}}, P\right)=i\left(T, P_{R_{1}}, P\right)-i\left(T, P_{r_{1}}, P\right)=0-1=-1 .
$$

Thus $T$ has at least one positive fixed point $y^{*}$ in $P_{r_{1}, R_{1}}$, which means that problem (1.1) has at least one positive solution $y^{*}$ satisfying $r_{1} \leq y^{*} \leq R_{1}$. This completes the proof.

Theorem 3.3 Suppose that conditions $\left(\mathrm{A}_{1}\right)$ and $\left(\mathrm{A}_{2}\right)$ hold. In addition, assume that the following conditions hold:

$\left(\mathrm{H}_{3}\right)\left(\frac{\omega^{*}}{v}\right)^{p-1}<g_{0}<+\infty$,

$\left(\mathrm{H}_{4}\right) \quad 0<g^{\infty}<\left(\frac{\omega_{*}}{2}\right)^{p-1}$.

Then problem (1.1) has at least one positive solution.

Proof From $\left(\mathrm{H}_{3}\right)$, for any $0<\varepsilon<g_{0}-\left(\frac{\omega^{*}}{v}\right)^{p-1}$, there exists a sufficiently large positive constant $r_{2}$ such that for any $t \in[0,1]$ and $0<y \leq r_{2}$, we have

$$
g(t, y) \geq\left(g_{0}-\varepsilon\right) y^{p-1}>\left(\frac{\omega^{*}}{v}\right)^{p-1} y^{p-1}, \quad(t, y) \in[0,1] \times\left(0, r_{2}\right]
$$


Thus, for any $y \in \partial P_{r_{2}}$, we get

$$
g(t, y) \geq\left(\frac{\omega^{*}}{v}\right)^{p-1} y^{p-1} \geq\left(\omega^{*} r_{2}\right)^{p-1}, \quad(t, y) \in[0,1] \times\left(0, r_{2}\right]
$$

Let $\varphi \equiv 1$. Now we prove that

$$
y \neq T y+\mu \varphi \quad \text { for any } y \in \partial P_{r_{2}} \text { and } \mu \geq 0
$$

Otherwise, there exist $y_{0} \in \partial P_{r_{2}}$ and $\mu_{0}>0$ such that $y_{0}=T y_{0}+\mu_{0} \varphi$. Let $\zeta=\min \left\{y_{0}(t)\right.$ : $t \in[v, 1-v]\}$, then for any $t \in[v, 1-v]$ and $0<t<\sigma^{*}$, we have

$$
\begin{aligned}
y_{0}= & T y_{0}(t)+\mu_{0} \varphi(t) \\
= & \phi_{q}\left(\frac{\beta_{1}}{\lambda_{1}} \phi_{p}\left(\int_{0}^{\sigma^{*}} \phi_{q}\left(\int_{0}^{s} b(r) g\left(r, y_{0}(r)\right) d r\right) d s\right)\right) \\
& +\int_{0}^{t} \int_{s}^{\sigma^{*}} \phi_{q}\left(\int_{0}^{r} b(x) g\left(x, y_{0}(x)\right) d x\right) d r d s+\mu_{0} \\
\geq & \int_{0}^{t} \int_{s}^{\sigma^{*}} \phi_{q}\left(\int_{0}^{r} b(x) g\left(x, y_{0}(x)\right) d x\right) d r d s+\mu_{0} \\
\geq & \int_{0}^{t} \int_{s}^{\sigma^{*}} \phi_{q}\left(\int_{0}^{r} b(x)\left(\frac{\omega^{*} y_{0}(x)}{v}\right)^{p-1} d x\right) d r d s+\mu_{0} \\
\geq & \frac{\omega^{*} v\left\|y_{0}\right\|}{v} \int_{v}^{t} \int_{s}^{\sigma^{*}} \phi_{q}\left(\int_{0}^{r} b(x) d x\right) d r d s+\mu_{0} \\
> & \omega^{*} \cdot \zeta \cdot f(t)+\mu_{0} \geq \omega^{*} \cdot \zeta \cdot m+\mu_{0}>\zeta+\mu_{0}>\zeta .
\end{aligned}
$$

On the other hand, by the same argument, for $t \in\left[\sigma^{*}, 1\right]$ and $t \in[v, 1-v]$, from (3.9), we have

$$
\begin{aligned}
y_{0}= & T y_{0}(t)+\mu_{0} \varphi(t) \\
= & \phi_{q}\left(\frac{\beta_{2}}{\lambda_{2}} \phi_{p}\left(\int_{\sigma^{*}}^{1} \phi_{q}\left(\int_{0}^{s} b(r) g\left(r, y_{0}(r)\right) d r\right) d s\right)\right) \\
& +\int_{t}^{1} \int_{\sigma^{*}}^{s} \phi_{q}\left(\int_{0}^{r} b(x) g\left(x, y_{0}(x)\right) d x\right) d r d s+\mu_{0} \\
\geq & \int_{t}^{1} \int_{\sigma^{*}}^{s} \phi_{q}\left(\int_{0}^{r} b(x) g\left(x, y_{0}(x)\right) d x\right) d r d s+\mu_{0} \\
\geq & \int_{t}^{1} \int_{\sigma^{*}}^{s} \phi_{q}\left(\int_{0}^{r} b(x)\left(\frac{\omega^{*} y_{0}(x)}{v}\right)^{p-1} d x\right) d r d s+\mu_{0} \\
\geq & \frac{\omega^{*} v\left\|y_{0}\right\|}{v} \int_{t}^{1-v} \int_{\sigma^{*}}^{s} \phi_{q}\left(\int_{0}^{r} b(x) d x\right) d r d s+\mu_{0} \\
> & \omega^{*} \zeta \cdot f(t)+\mu_{0}>\omega^{*} \cdot \zeta \cdot m+\mu_{0}>\zeta+\mu_{0}>\zeta .
\end{aligned}
$$

From (3.15) and (3.16), we easily obtain $\zeta>\zeta$, a contradiction with the definition of $\zeta$. From the above discussion we see that (3.14) holds. It follows from Lemma 2.2 that

$$
i\left(T, P_{r_{2}}, P\right)=0
$$


Next from $\left(\mathrm{H}_{4}\right)$, for any $0<\varepsilon_{1}<\left(\frac{\omega_{*}}{2}\right)^{p-1}-g^{\infty}$, there exists a sufficiently small positive constant $R_{*}$ such that $y \geq R_{*}, t \in[0,1]$, we have

$$
g(t, y) \leq y^{p-1}\left(g_{\infty}+\varepsilon\right)<y^{p-1}\left(\frac{\omega_{*}}{2}\right)^{p-1}, \quad(t, y) \in[0,1] \times\left[R_{*},+\infty\right) .
$$

Let $M_{0}=\sup _{y \in \partial P_{R_{*}}} \int_{0}^{1} b(s) g(s, y(s)) d s$. Then $M_{0}<+\infty$ by $(2.12)$. Take

$$
R_{2}>\max \left\{R_{*}, 2 r_{2},\left(\frac{2}{\omega_{*}}\right)\left(\frac{M_{0}}{\int_{0}^{1} b(r) d r}\right)^{\frac{1}{p-1}}\right\} .
$$

Notice $y \in \partial P_{R_{*}}$ implies that $y(t) \leq\|y\| \leq R_{*}$. In addition, for any $y \in \partial P_{R_{2}}$, let $D[y]=\{t \in$ $\left.[0,1]: y \geq R_{*}\right\}$, then for any $t \in D[y]$, clearly, $R_{*} \leq y \leq\|y\|=R_{2}$. Let $y_{1}(t)=\min \left\{y(t), R_{*}\right\}$, then $y_{1} \in \partial P_{R_{*}}$. Thus, for any $y \in \partial P_{R_{2}}$, from (3.7) and (3.18), we have

$$
\begin{aligned}
\|T y\|= & \max _{0 \leq t \leq 1}(T y)(t)=(T y)\left(\sigma^{*}\right) \\
\leq & \phi_{q}\left(\frac{\beta_{i}}{\lambda_{i}} \phi_{p}\left(\int_{0}^{1} \phi_{q}\left(\int_{0}^{1} b(r) g(r, y(r)) d r\right) d s\right)\right) \\
& +\int_{0}^{1} \int_{0}^{1} \phi_{q}\left(\int_{0}^{1} b(x) g(x, y(x)) d x\right) d r d s \\
= & \phi_{q}\left(\frac{\beta_{i}}{\lambda_{i}} \int_{0}^{1} b(r) g(r, y(r)) d r\right)+\phi_{q}\left(\int_{0}^{1} b(r) g(r, y(r)) d r\right) \\
\leq & \left(1+\phi_{q}\left(\frac{\beta_{i}}{\lambda_{i}}\right)\right) \phi_{q}\left(\int_{0}^{1} b(r) g(r, y(r)) d r\right) \\
\leq & \left(1+\phi_{q}\left(\frac{\beta_{i}}{\lambda_{i}}\right)\right) \phi_{q}\left(\int_{D[y]} b(r) g(r, y(r)) d r+\int_{[0,1] \backslash D[y]} b(r) g(r, y(r)) d r\right) \\
\leq & \left(1+\phi_{q}\left(\frac{\beta_{i}}{\lambda_{i}}\right)\right) \phi_{q}\left(\int_{0}^{1} b(r)\left(\frac{\omega_{*}}{2} y(r)\right)^{p-1} d r+\int_{0}^{1} b(r) g(r, y(r)) d r\right) \\
\leq & \left(1+\phi_{q}\left(\frac{\beta_{i}}{\lambda_{i}}\right)\right) \phi_{q}\left(\int_{0}^{1} b(r)\left(\frac{\omega_{*}}{2} y(r)\right)^{p-1} d r+M_{0}\right) \\
\leq & \left(1+\phi_{q}\left(\frac{\beta_{i}}{\lambda_{i}}\right)\right) \phi_{q}\left(\left(\frac{\omega_{*}}{2} R_{2}\right)^{p-1} \int_{0}^{1} b(r) d r+\left(\frac{\omega_{*}}{2} R_{2}\right)^{p-1} \int_{0}^{1} b(r) d r\right) \\
= & \left(1+\phi_{q}\left(\frac{\beta_{i}}{\lambda_{i}}\right)\right) \phi_{q}\left(\left(\frac{\omega_{*}}{2} R_{2}\right)^{p-1} 2 \int_{0}^{1} b(r) d r\right) \\
= & \left(1+\phi_{q}\left(\frac{\beta_{i}}{\lambda_{i}}\right)\right) \phi_{q}\left(2 \int_{0}^{1} b(r) d r\right) \frac{\omega_{*}}{2} R_{2}<\frac{R_{2}}{2}<R_{2}=\|y\| \quad \text { for } i=1,2 . \\
& \\
&
\end{aligned}
$$

Therefore $\|T y\|<\|y\|$ for any $y \in \partial P_{R_{2}}$. It follows from Lemma 2.4 that

$$
i\left(T, P_{R_{2}}, P\right)=1 \text {. }
$$

From (3.17) and (3.19) together with the properties of fixed point index, we have

$$
i\left(T, P_{r_{2}, R_{2}}, P\right)=i\left(T, P_{R_{2}}, P\right)-i\left(T, P_{r_{2}}, P\right)=1-0=1 .
$$


Thus $T$ has at least one positive fixed point $y^{* *}$ in $P_{r_{2}, R_{2}}$ with $r_{2} \leq y^{* *} \leq R_{2}$. Therefore $y^{* *}$ is a positive solution of problem (1.1). This completes the proof.

\section{The existence of multiple positive solutions}

Theorem 4.1 Suppose that conditions $\left(\mathrm{A}_{1}\right)$ and $\left(\mathrm{A}_{2}\right)$ hold. In addition, $\left(\mathrm{H}_{1}\right)$ and $\left(\mathrm{H}_{4}\right)$ hold, and there exists a positive constant $r_{0}^{*}>0$ such that

$$
g(t, y) \geq\left(l r_{0}^{*}\right)^{p-1}, \quad v r_{0}^{*} \leq y \leq r_{0}^{*}, 0<t<1
$$

Then problem (1.1) has at least two positive solutions.

Proof It follows from $\left(\mathrm{H}_{1}\right)$ that there exists a positive constant $r_{1}^{*}$ satisfying $0<r_{1}^{*} \leq r_{0}^{*}$ such that

$$
g(t, y) \leq\left(\frac{\omega_{*}}{2} y\right)^{p-1}, \quad 0<y \leq r_{1}^{*}, 0 \leq t \leq 1
$$

By making use of $\left(\mathrm{H}_{4}\right)$, there exists a positive constant $r_{2}^{*}$ satisfying $r_{2}^{*}>r_{0}^{*}>0$ such that

$$
g(t, y) \leq\left(\frac{\omega_{*}}{2} y\right)^{p-1}, \quad y \geq r_{2}^{*}, 0 \leq t \leq 1
$$

Without loss of generality, we may assume that $T$ has no fixed point on $\partial P_{r_{1}^{*}}$ and $\partial P_{r_{2}^{*}}$. From (4.2)-(4.3) with the corresponding proofs in Theorems 3.2 and 3.3, respectively, together with the permanence property of fixed point index, we have

$$
i\left(T, P_{r_{1}^{*}}, P\right)=1
$$

and

$$
i\left(T, P_{r_{2}^{*}}, P\right)=1
$$

For any $y \in \partial P_{r_{0}^{*}}$, by the concavity of function $y(t)$ for $t \in[0,1]$, we easily get $0<v\|y\| \leq$ $y(t) \leq\|y\|=r_{0}^{*}, t \in[0,1]$. Thus, for any $y \in \partial P_{r_{0}^{*}}$, in view of (4.1) and the corresponding proof in Theorem 3.1 together with the permanence property of fixed point index, we have

$$
i\left(T, P_{r_{0}^{*}}, P\right)=0
$$

From (4.4) and (4.6) together with the properties of fixed point index, we obtain

$$
\begin{aligned}
& i\left(T, P_{r_{1}^{*}, r_{0}^{*}}, P\right)=i\left(T, P_{r_{0}^{*}}, P\right)-i\left(T, P_{r_{1}^{*}}, P\right)=0-1=-1, \\
& i\left(T, P_{r_{0}^{*}, r_{2}^{*}}, P\right)=i\left(T, P_{r_{2}^{*}}, P\right)-i\left(T, P_{r_{0}^{*}}, P\right)=1-0=1 .
\end{aligned}
$$

Hence, $T$ has at least two positive fixed points in $P_{r_{1}^{*}, r_{0}^{*}}$ and $P_{r_{0}^{*}}, r_{2}^{*}$, respectively. Therefore, problem (1.1) has at least two positive solutions. This completes the proof. 
Theorem 4.2 Suppose that conditions $\left(\mathrm{A}_{1}\right)$ and $\left(\mathrm{A}_{2}\right)$ hold. In addition, $\left(\mathrm{H}_{2}\right)$ and $\left(\mathrm{H}_{3}\right)$ hold, and there exists a positive constant $\tilde{r}_{0}^{*}>\sqrt{\Gamma_{1}}$ such that

$$
g(t, y) \leq\left(\frac{v(1-v)}{y}\right)^{p-1}, \quad 0<y(t) \leq \tilde{r}_{0}^{*}, 0<t<1,
$$

where $\Gamma_{1}=\max \left\{\left(1+\phi_{q}\left(\frac{\beta_{1}}{\lambda_{1}}\right)\right) \phi_{q}\left(2 \int_{0}^{1} b(r) d r\right),\left(1+\phi_{q}\left(\frac{\beta_{2}}{\lambda_{2}}\right)\right) \phi_{q}\left(2 \int_{0}^{1} b(r) d r\right)\right\}$.

Then problem (1.1) has at least two positive solutions.

Proof It follows from $\left(\mathrm{H}_{3}\right)$ that there exists a positive constant $\tilde{r}_{1}^{*}>0$ satisfying $0<\tilde{r}_{1}^{*}<$ $\sqrt{\Gamma_{1}}$ such that

$$
g(t, y) \geq\left(\frac{\omega_{*}}{v} y\right)^{p-1}, \quad 0<y \leq \tilde{r}_{1}^{*}, 0 \leq t \leq 1 .
$$

By making use of $\left(\mathrm{H}_{2}\right)$, there exists a positive constant $\tilde{r}_{3}^{*}>\tilde{r}_{0}^{*}>0$ such that

$$
g(t, y) \geq\left(\frac{\omega_{*}}{v} y\right)^{p-1}, \quad y \geq v \tilde{r}_{3}^{*}, 0 \leq t \leq 1
$$

Then, for any $y \in \partial P_{r_{3}^{*}}$, we have

$$
g(t, y) \geq\left(\frac{\omega_{*}}{v} y\right)^{p-1}, \quad y \geq v \tilde{r}_{3}^{*}=v\|y\|, t \in[v, 1-v]
$$

It follows from Lemma 2.6 that $T: \bar{P}_{r_{1}^{*}, r_{3}^{*}} \longrightarrow P$ is a completely continuous operator. Now we extend the operator $T$, still denoted by $T$, then $T: \bar{P}_{\tilde{r}_{3}^{*}} \rightarrow P$ is completely continuous. Without loss of generality, we may assume that $T$ has no fixed point on $\partial P_{r_{1}^{*}}$ and $\partial P_{r_{3}^{*}}$. From (4.8) and (4.9), the corresponding proofs in Theorems 3.2 and 3.3, respectively, together with the permanence property of fixed point index, we have

$$
i\left(T, P_{\tilde{r}_{1}^{*}}, P\right)=0
$$

and

$$
i\left(T, P_{\tilde{r}_{3}^{*}}, P\right)=0 .
$$

Choose $\sqrt{\Gamma_{1}}<\tilde{r}_{2}^{*} \leq \tilde{r}_{0}^{*}$. For all $y \in \partial P_{r_{2}^{*}}$, by the concavity of function $y(t)$ on $(0,1)$, we see $y(t) \geq v\|y\|$ for $t \in(0,1)$ and $0<y(t) \leq \tilde{r}_{2}^{*} \leq \tilde{r}_{0}^{*}$ for $t \in(0,1)$. It follows from (4.7) and $y \in \partial P_{r_{2}^{*}}$ that

$$
g(t, y(t)) \leq\left(\frac{v(1-v)}{y(t)}\right)^{p-1} \leq\left(\frac{v(1-v)}{\|y\| v}\right)^{p-1}<\frac{1}{\tilde{r}_{2}^{* p-1}} \quad \text { for } t \in(0,1) .
$$

Therefore, for any $y \in \partial P_{\tilde{r}_{2}^{*}}$, we obtain

$$
\begin{aligned}
\|T y\| & =\max _{0 \leq t \leq 1}(T y)(t)=(T y)\left(\sigma^{*}\right) \\
& \leq \phi_{q}\left(\frac{\beta_{i}}{\lambda_{i}} \phi_{p}\left(\int_{0}^{1} \phi_{q}\left(\int_{0}^{1} b(r) g(r, y(r)) d r\right) d s\right)\right)
\end{aligned}
$$




$$
\begin{aligned}
& +\int_{0}^{1} \int_{0}^{1} \phi_{q}\left(\int_{0}^{1} b(x) g(x, y(x)) d x\right) d r d s \\
= & \phi_{q}\left(\frac{\beta_{i}}{\lambda_{i}} \int_{0}^{1} b(r) g(r, y(r)) d r\right)+\phi_{q}\left(\int_{0}^{1} b(r) g(r, y(r)) d r\right) \\
\leq & \left(1+\phi_{q}\left(\frac{\beta_{i}}{\lambda_{i}}\right)\right) \phi_{q}\left(\int_{0}^{1} b(r) g(r, y(r)) d r\right) \\
< & \left(1+\phi_{q}\left(\frac{\beta_{i}}{\lambda_{i}}\right)\right) \frac{1}{\tilde{r}_{2}^{*}} \phi_{q}\left(\int_{0}^{1} b(r) d r\right) \\
= & \frac{1}{\tilde{r}_{2}^{*}}\left(1+\phi_{q}\left(\frac{\beta_{i}}{\lambda_{i}}\right)\right) \phi_{q}\left(\int_{0}^{1} b(r) d r\right) \\
< & \frac{1}{\tilde{r}_{2}^{*}} \cdot \Gamma_{1}<\tilde{r}_{2}^{*}=\|y\| \quad \text { for } i=1,2 .
\end{aligned}
$$

Thus $\|T y\|<\|y\|$ for any $y \in \partial P_{r_{2}^{*}}$. From Lemma 2.5 we have

$$
i\left(T, P_{\widetilde{r}_{2}^{*}}, P\right)=1
$$

By making use of (4.10) and (4.11) with (4.12), combining the properties of fixed point index, we have

$$
\begin{aligned}
& i\left(T, P_{\tilde{r}_{1}^{*}, r_{2}^{*}}, P\right)=i\left(T, P_{r_{2}^{*}}, P\right)-i\left(T, P_{r_{1}^{*}}, P\right)=1-0=1, \\
& i\left(T, P_{\tilde{r}_{2}^{*}, r_{3}^{*}}, P\right)=i\left(T, P_{r_{3}^{*}}, P\right)-i\left(T, P_{r_{2}^{*}}, P\right)=0-1=-1 .
\end{aligned}
$$

Hence, $T$ has at least two positive fixed points in $P_{r_{1}^{*}, r_{2}^{*}}$ and $P_{r_{r}^{*}, r_{3}^{*}}$, respectively. Therefore, problem (1.1) has at least two positive solutions. This completes the proof.

\section{The existence of infinite positive solutions}

Now we shall discuss the existence of infinitely many positive solutions. For convenience, we make the following assumptions:

$\left(\mathrm{A}_{1}^{\prime}\right)$ There exists a nonincreasing sequence $\left\{t_{n}\right\}_{n=1}^{\infty}$ such that $0<t_{n+1}<t_{n}, t_{1}<\frac{1}{2}$, $\lim _{n \rightarrow \infty} t_{n}=t_{0} \geq 0$, with $\lim _{t \rightarrow t_{n}} b(t)=\infty, n=1,2, \ldots$, and $0<\int_{0}^{1} b(t) d t<+\infty$. Moreover, $b(t)$ does not vanish identical on any subinterval of $[0,1]$.

Theorem 5.1 Suppose that conditions $\left(\mathrm{A}_{1}^{\prime}\right)$ and $\left(\mathrm{A}_{2}\right)$ hold. Let $\left\{v_{k}\right\}_{k=1}^{\infty}$ be such that $v_{k} \in$ $\left(t_{k+1}, t_{k}\right)(k=1,2, \ldots)$. Let $\left\{r_{k}\right\}_{k=1}^{\infty}$ and $\left\{R_{k}\right\}_{k=1}^{\infty}$ be such that $v_{k} r_{k}<r_{k}<R_{k}, k=1,2, \ldots$. For each natural number $k$, we assume that $g$ satisfies

(E $\left.\mathrm{E}_{1}\right) g(t, y) \geq\left(l r_{k}\right)^{p-1},(t, y) \in[0,1] \times\left[v_{k} r_{k}, R_{k}\right]$;

(E $\left.\mathrm{E}_{2}\right) g(t, y) \leq\left(L R_{k}\right)^{p-1},(t, y) \in[0,1] \times\left(0, R_{k}\right]$.

Then boundary value problem (1.1) has infinitely many positive solutions $\left\{y_{k}\right\}_{k=1}^{\infty}$ such that $\left\|y_{k}\right\|$ is between $r_{k}$ and $R_{k}, k=1,2, \ldots$.

Proof From $\left(\mathrm{A}_{1}^{\prime}\right)$, we know that $t_{0}<t_{k+1}<v_{k}<t_{k}<\frac{1}{2}, k=1,2, \ldots$, then for any $k \in N, y \in P$, we have

$$
y(t) \geq v_{k}\|y\|, \quad t \in\left[v_{k}, 1-v_{k}\right] .
$$


We easily know that $\left\{P_{r_{k}}\right\}_{k=1}^{\infty}$ and $\left\{P_{R_{k}}\right\}_{k=1}^{\infty}$ are subsets of $P$. For a fixed $k$ and for any $y \in P_{r_{k}}$, from (5.1) we have $y(t) \geq v_{k}\|y\|=v_{k} r_{k}, t \in\left[v_{k}, 1-v_{k}\right]$. For $\left[t_{1}, 1-t_{1}\right] \subset\left[v_{k}, 1-v_{k}\right] \subset[0,1]$, let $\psi \equiv 1$. We prove that

$$
y \neq T y+\mu \psi \quad \text { for } y \in \partial P_{r_{k}} \text { and } \mu \geq 0 .
$$

If not, there exist $\tilde{y}_{0} \in \partial P_{r_{k}}$ and $\mu_{0} \geq 0$ such that $\tilde{y}_{0}=T \tilde{y}_{0}+\mu_{0} \psi$. Let $\mu^{*}=\min _{t \in[0,1]} \tilde{y}_{0}(t)$. Then, for $t \in\left(0, \sigma^{*}\right)$, we have

$$
\begin{aligned}
\tilde{y}_{0}(t) & =T \tilde{y}_{0}(t)+\mu_{0} \\
& \geq \int_{0}^{t} \int_{s}^{\sigma^{*}} \phi_{q}\left(\int_{0}^{r} b(x) g\left(x, \tilde{y}_{0}(x)\right) d x\right) d r d s+\mu_{0} \\
& \geq\left(l r_{k}\right) \int_{0}^{t} \int_{s}^{\sigma^{*}} \phi_{q}\left(\int_{0}^{r} b(x) d x\right) d r d s+\mu_{0} \\
& \geq\left(l r_{k}\right) \int_{v_{k}}^{t} \int_{s}^{\sigma^{*}} \phi_{q}\left(\int_{0}^{r} b(x) d x\right) d r d s+\mu_{0} \\
& \geq\left(l r_{k}\right) \int_{t_{1}}^{t} \int_{s}^{\sigma^{*}} \phi_{q}\left(\int_{0}^{r} b(x) d x\right) d r d s+\mu_{0} \\
& \geq\left(l r_{k}\right) f(t)+\mu_{0} \geq\left(l r_{k}\right) m+\mu_{0}>r_{k}=\left\|\tilde{y}_{0}\right\|>\mu^{*} .
\end{aligned}
$$

On the other hand, for $t \in\left[\sigma^{*}, 1\right]$, by the same argument, we have

$$
\begin{aligned}
\tilde{y}_{0}(t) & =T \tilde{y}_{0}(t)+\mu_{0} \\
& \geq \int_{t}^{1} \int_{\sigma^{*}}^{s} \phi_{q}\left(\int_{0}^{r} b(x) g\left(x, \tilde{y}_{0}(x)\right) d x\right) d r d s+\mu_{0} \\
& \geq\left(l r_{k}\right) \int_{t}^{1} \int_{\sigma^{*}}^{s} \phi_{q}\left(\int_{0}^{r} b(x) d x\right) d r d s+\mu_{0} \\
& \geq\left(l r_{k}\right) \int_{t}^{1-v_{k}} \int_{\sigma^{*}}^{s} \phi_{q}\left(\int_{0}^{r} b(x) d x\right) d r d s+\mu_{0} \\
& \geq\left(l r_{k}\right) \int_{t}^{1-t_{1}} \int_{\sigma^{*}}^{s} \phi_{q}\left(\int_{0}^{r} b(x) d x\right) d r d s+\mu_{0} \\
& \geq\left(l r_{k}\right) f(t)+\mu_{0} \geq\left(l r_{k}\right) m+\mu_{0}>r_{k}=\left\|\tilde{y}_{0}\right\|>\mu^{*} .
\end{aligned}
$$

From the above discussions, we see $\tilde{y}_{0}>\mu^{*}$, which implies $\mu^{*}>\mu^{*}$, a contradiction with the definition of $\mu^{*}$. Thus, we know that (5.2) holds. It follows from Lemma 2.2 that

$$
i\left(T, P_{r_{k}}, P\right)=0 .
$$

Without loss of generality, we may assume that $T$ has no fixed point on $\partial P_{r_{k}}$ and $\partial P_{R_{k}}$. Now we prove that

$$
T y \neq \eta y, \quad \forall y \in \partial P_{R_{k}}, \eta \geq 1 .
$$


In fact, if not, there exist $\tilde{y}_{1} \in \partial P_{R_{k}}$ and $\tilde{\eta}_{1} \geq 1$ such that $T \tilde{y}_{1}=\tilde{\eta}_{1} \tilde{y}_{1}$. Let $\tilde{y}_{1}^{*}(t)=$ $\min \left\{\tilde{y}_{1}(t), R_{k}\right\}$, thus $\tilde{y}_{1}^{*} \in \partial P_{R_{k}}$. Thus, for $t \in\left(0, \sigma^{*}\right)$, we have

$$
\begin{aligned}
\tilde{y}_{1} \leq & \phi_{q}\left(\frac{\beta_{1}}{\lambda_{1}} \int_{0}^{1} b(r) g\left(r, \tilde{y}_{1}(r)\right) d r\right) \\
& +\phi_{q}\left(\int_{0}^{1} b(r) g\left(r, \tilde{y}_{1}(r)\right) d r\right) \\
= & \left(1+\phi_{q}\left(\frac{\beta_{1}}{\lambda_{1}}\right)\right) \int_{0}^{1} b(r) g\left(r, \tilde{y}_{1}(r)\right) d r \\
\leq & L R_{k}\left(1+\phi_{q}\left(\frac{\beta_{1}}{\lambda_{1}}\right)\right) \phi_{q}\left(\int_{0}^{1} b(r) d r\right) \\
< & \omega_{*} \cdot R_{k} \cdot \frac{1}{\omega_{*}}=R_{k}=\left\|\tilde{y}_{1}^{*}\right\| .
\end{aligned}
$$

On the other hand, for $t \in\left[\sigma^{*}, 1\right]$, by the same argument, we have

$$
\begin{aligned}
\tilde{y}_{1} \leq & \phi_{q}\left(\frac{\beta_{2}}{\lambda_{2}} \int_{0}^{1} b(r) g\left(r, \tilde{y}_{1}(r)\right) d r\right) \\
& +\phi_{q}\left(\int_{0}^{1} b(r) g\left(r, \tilde{y}_{1}(r)\right) d r\right) \\
= & \left(1+\phi_{q}\left(\frac{\beta_{2}}{\lambda_{2}}\right)\right) \int_{0}^{1} b(r) g\left(r, \tilde{y}_{1}(r)\right) d r \\
\leq & L R_{k}\left(1+\phi_{q}\left(\frac{\beta_{2}}{\lambda_{2}}\right)\right) \phi_{q}\left(\int_{0}^{1} b(r) d r\right) \\
< & \omega_{*} \cdot R_{k} \cdot \frac{1}{\omega_{*}}=R_{k}=\left\|\tilde{y}_{1}^{*}\right\| .
\end{aligned}
$$

The above discussions imply that $\left\|\tilde{y}_{1}^{*}\right\|<\left\|\tilde{y}_{1}^{*}\right\|$, a contradiction with the definition of $\tilde{y}_{1}^{*}$. Therefore (5.4) holds. It follows from Lemma 2.3 that

$$
i\left(T, P_{R_{k}}, P\right)=1 .
$$

From (5.3) and (5.5) together with the properties of fixed point index, we have

$$
i\left(T, P_{r_{k}, R_{k}}, P\right)=i\left(T, P_{R_{k}}, P\right)-i\left(T, P_{r_{k}}, P\right)=1-0=1
$$

Thus $T$ has at least one positive fixed point $y_{k}$ in $P_{r_{k}, R_{k}}$, which means that problem (1.1) has at least one positive solution $y_{k}$ satisfying $r_{k} \leq y_{k} \leq R_{k}$. From the randomness of $k$ we see that Theorem 5.1 holds. This completes the proof.

Theorem 5.2 Suppose that conditions $\left(\mathrm{A}_{1}^{\prime}\right)$ and $\left(\mathrm{A}_{2}\right)$ hold. Let $\left\{v_{k}\right\}_{k=1}^{\infty}$ be such that $v_{k} \in$ $\left(t_{k+1}, t_{k}\right)(k=1,2, \ldots)$. Let $\left\{r_{k}\right\}_{k=1}^{\infty}$ and $\left\{R_{k}\right\}_{k=1}^{\infty}$ be such that $v_{k} r_{k}<r_{k}<R_{k}, k=1,2, \ldots$. For each natural number $k$, we assume that $g$ satisfies

(E $\left.\mathrm{E}_{3}\right) \quad 0<g^{0}<\left(\frac{\omega_{*}}{2}\right)^{p-1}$,

$\left(\mathrm{E}_{4}\right)\left(\frac{\omega^{*}}{v_{k}}\right)^{p-1}<g_{\infty}<+\infty$. 
Then boundary value problem (1.1) has infinitely many positive solutions $\left\{y_{k}\right\}_{k=1}^{\infty}$ such that $\left\|y_{k}\right\|$ is between $r_{k}$ and $R_{k}, k=1,2, \ldots$.

Theorem 5.3 Suppose that conditions $\left(\mathrm{A}_{1}^{\prime}\right)$ and $\left(\mathrm{A}_{2}\right)$ hold. Let $\left\{v_{k}\right\}_{k=1}^{\infty}$ be such that $v_{k} \in$ $\left(t_{k+1}, t_{k}\right)(k=1,2, \ldots)$. Let $\left\{r_{k}\right\}_{k=1}^{\infty}$ and $\left\{R_{k}\right\}_{k=1}^{\infty}$ be such that $v_{k} r_{k}<r_{k}<R_{k}, k=1,2, \ldots$ For each natural number $k$, we assume that $g$ satisfies

( $\left.\mathrm{E}_{5}\right)\left(\frac{\omega^{*}}{v_{k}}\right)^{p-1}<g_{0}<+\infty$,

( $\left.\mathrm{E}_{6}\right) \quad 0<g^{\infty}<\left(\frac{\omega_{*}}{2}\right)^{p-1}$.

Then boundary value problem (1.1) has infinitely many positive solutions $\left\{y_{k}\right\}_{k=1}^{\infty}$ such that $\left\|y_{k}\right\|$ is between $r_{k}$ and $R_{k}, k=1,2, \ldots$.

\section{Competing interests}

The author declares that she has no competing interests.

\section{Acknowledgements}

The author is very grateful to the reviewers for their thorough reading of the manuscript and for their valuable comments and excellent suggestions. She would like to express her gratitude to Professor Lishan Liu and Professor RP Agarwal for their many valuable comments. The author was supported financially by the Foundation of Shanghai Natural Science (13ZR1430100) and the NNSF of P.R. China (No. 11371095, No. 2013M541455).

\section{Publisher's Note}

Springer Nature remains neutral with regard to jurisdictional claims in published maps and institutional affiliations.

Received: 6 January 2017 Accepted: 21 March 2017 Published online: 29 March 2017

\section{References}

1. Chen, S: The existence of multiple positive solutions for a class of third-order $p$-Laplacian operator singular boundary value problems. Acta Math. Sci. Ser. A 26(5), 794-800 (2006) (in Chinese)

2. He, X: Double positive solutions of a three-point boundary value problem for the one-dimensional $p$-Laplacian. Appl. Math. Lett. 17, 867-873 (2004)

3. $\mathrm{He}, \mathrm{X}, \mathrm{Ge}, \mathrm{W}$ : Twin positive solutions for the one dimensional $p$-Laplacian boundary value problems. Nonlinear Anal. $56,975-984$ (2004)

4. Gupta, C, Trofimchuk, S: A sharper condition for the solvability of a three-point second order boundary value problem. J. Math. Anal. Appl. 205, 586-597 (1997)

5. Kong, L, Wang, J: Multiple positive solutions for the one-dimensional p-Laplacian. Nonlinear Anal. 42, 1327-1333 (2000)

6. Liu, B: Positive solutions of three-point boundary value problems for the one-dimensional $p$-Laplacian with infinitely many singularities. Appl. Math. Lett. 17, 655-661 (2004)

7. Kong, D, Liu, L, Wu, Y: Triple positive solutions of a boundary value problem for nonlinear singular second-order differential equations of mixed type with $p$-Laplacian. Comput. Math. Appl. 58, 1425-1432 (2009)

8. Su, H, Wei, Z, Xu, F: The existence of positive solutions for nonlinear singular boundary value system with $p$-Laplacian. Appl. Math. Comput. 181, 826-836 (2006)

9. Yang, C, Yan, J: Positive solutions for third-order Sturm-Liouville boundary value problems with p-Laplacian. Comput. Math. Appl. 59, 2059-2066 (2010)

10. Yang, C, Zhai, C, Yan, J: Positive solutions of the three-point boundary value problem for second order differential equations with an advanced argument. Nonlinear Anal. 65, 2013-2023 (2006)

11. Feng, W, Webb, JRL: Solvability of $m$-point boundary value problems with nonlinear growth. J. Math. Anal. Appl. 212, 467-480 (1997)

12. Deimling, K: Nonlinear Functional Analysis. Springer, New York (1985)

13. Guo, D, Lakshmikantham, V: Nonlinear Problems in Abstract Cones. Academic Press, Boston (1988)

14. Ma, D, Du, Z, Ge, W: Existence and iteration of monotone positive solutions for multipoint boundary value problem with $p$-Laplacian operator. Comput. Math. Appl. 50, 729-739 (2005)

15. Li, X: Multiple positive solutions for some four-point boundary value problems with $p$-Laplacian. Appl. Math. Comput. 202, 413-426 (2008)

16. Lan, K, Webb, JRL: Positive solutions of semilinear differential equations with singularities. J. Differ. Equ. 148, 407-421 (1998)

17. Ji, D, Ge, W: Multiple positive solutions for some $p$-Laplacian boundary value problems. Appl. Math. Comput. 187 $1315-1325$ (2007)

18. Su, H, Wei, Z, Wang, B: The existence of positive solutions for a nonlinear four-point singular boundary value problem with a $p$-Laplacian operator. Nonlinear Anal. 66, 2204-2217 (2007)

19. Sun, Y, Liu, L, Zhang, J, Agarwal, RP: Positive solutions of singular three-point boundary value problems for second-order differential equations. J. Comput. Appl. Math. 230(1), 738-750 (2009) 
20. Wang, Y, Hou, C: Existence of multiple positive solutions for one-dimensional p-Laplacian. J. Math. Anal. Appl. 315 144-153 (2006)

21. Wang, Y, Ge, W: Multiple positive solutions for multipoint boundary value problems with one-dimensional p-Laplacian. J. Math. Anal. Appl. 327, 1381-1395 (2007)

22. Wang, Z, Zhang, J: Positive solutions for one-dimensional $p$-Laplacian boundary value problems with dependence on the first order derivative. J. Math. Anal. Appl. 314, 618-630 (2006)

23. Zhao, D, Wang, $H, G e, W$ : Existence of triple positive solutions to a class of $p$-Laplacian boundary value problems. J. Math. Anal. Appl. 328, 972-983 (2007)

24. Zhang, G, Sun, J: Positive solutions of a class of singular boundary value problems. Acta Math. Appl. Sin. 29(2), 297-310 (2006) (in Chinese)

Submit your manuscript to a SpringerOpen ${ }^{\circ}$ journal and benefit from:

- Convenient online submission

Rigorous peer review

- Immediate publication on acceptance

- Open access: articles freely available online

- High visibility within the field

- Retaining the copyright to your article 